\title{
CLINICAL-EPIDEMIOLOGICAL ANALYSIS OF PATIENTS REFERRED TO A TERTIARY RHEUMATOLOGY SERVISSE
}

Juliana de Jesus Boscolo ^,^, Danielly Dantas Pimentel', Gizelle Gouvea Rezende', Gustavo Roberto Lourenço1', lane Tamara Dondé', Luciana Akita', Viviane Alves Costa', Viviane Queiroz de Oliveira Maia', Carolina Goulart de Carvalho², Taísa Morete da Silva'

1.Hospital de Base de São José do Rio Preto, São José do Rio Preto (SP), Brazil. 2.Centro Universitário de Santa Fé do Sul, Santa Fé do Sul (SP), Brazil.

*Corresponding author: julianaboscolo.jjb@gmail.com

\section{BACKGROUND}

The basic health care distinguishes itself as the gateway to the National Health Service (SUS), being responsible for the resolution of around $85 \%$ of health problems and for the referencing of higher complexity levels. The regulation of the assistance has the role of sorting the access to actions and to healthcare services, properly allocating patients with greater needs and clinical indication. This study aims to evaluate the epidemiological profile, origin and degree of diagnostic agreement of patients referred to a tertiary rheumatology service, as well as the waiting time until their medical appointments.

\section{MATERIALS AND METHODS}

This cross-sectional study evaluates the referral of admitted patients in a period of 12 weeks, between May and August of 2020, analyzing age, gender and waiting time. A comparative analysis of agreement will be made, between the referral diagnosis and the main diagnosis made by the medical team after their first evaluation.

\section{RESULTS}

64 referral forms were evaluated among patients with an average age of 53.4 years old in the moment of their first evaluation, $84.4 \%$ being females, with an average waiting time of 5.3 months between referral and first medical appointment. Rheumatoid arthritis was the prevalent diagnosis both in referral, with a total of $29 \mathrm{forms}$, and after the first evaluation done by the rheumatology team, with a total of 22 patients, although it was observed an increase in diagnosis of fibromyalgia, from 4 to 18 , after this first evaluation. The percentage of agreement among all referral was of $59.4 \%$, with $77.7 \%$ of agreement among patients referred from secondary healthcare services and $35.7 \%$ among those referred from primary healthcare services, a statistically meaningful difference $(p<0,01)$. The percentage of diagnostic agreement of patients according to their origin is shown in Fig. 1.

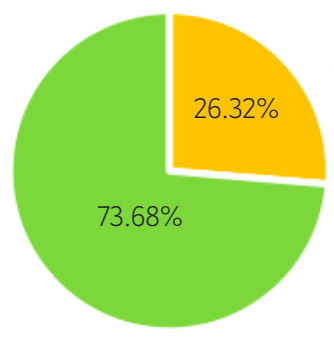

Primary healthcare services

Secondary healthcare services

Figure 1. Diagnostic agreement of patients according to the origin.

\section{CONCLUSION}

The epidemiological profile evaluated was predominantly feminine, on their 6th decade of life, with an average waiting time of nearly five months. Rheumatoid arthritis was the prevalent pathology among the referrals, with a general $59.4 \%$ degree of agreement. The secondary healthcare service presented a greater agreement between the referral diagnosis and the one given by the rheumatology after their first evaluation. 